\title{
Three-Body Hadron Resonances
}

\section{A. Martínez Torres*}

Departamento de Física Teórica and IFIC, Centro Mixto Universidad deValencia-CSIC, Institutos de Investigación de Paterna, Aptdo. 22085, 46071 Valencia, Spain.

E-mail: amartinedific.uv.es

\section{K. P. Khemchandani}

Centro de Física Teórica, Departamento de Física, Universidade de Coimbra, P-3004-516

Coimbra, Portugal.

E-mail: kanchandteor.fis.uc.pt

\section{E. Oset}

Departamento de Física Teórica and IFIC, Centro Mixto Universidad deValencia-CSIC, Institutos de Investigación de Paterna, Aptdo. 22085, 46071 Valencia, Spain.

E-mail: oset@ific.uv.es

We have studied different three-body hadron systems using the Faddeev equations combined with Unitary Chiral Dynamics in a coupled channel approach. In the strangeness $S=-1$ sector, we have solved the equations for the $\pi \bar{K} N$ system and its coupled channels and we report four $\Sigma$ 's and two $\Lambda$ 's, in the $1500-1800 \mathrm{MeV}$ region, as two meson-one baryon $\mathrm{S}$-wave $1 / 2^{+}$resonances. For the $S=0$ sector, we find resonances in the $\pi \pi N$ system at energies $1704-i 375 / 2 \mathrm{MeV}$, $2080-i 54 / 2 \mathrm{MeV}$ with total isospin $1 / 2$ and at 2126-i42/2 MeV with total isospin 3/2. All of them have $J^{\pi}=1 / 2^{+}$. We identify these states with the $N^{*}(1710)$, the $N^{*}(2100)$ and the $\Delta(1910)$, respectively, listed by the PDG. Apart from these, we obtain a peak with isospin $1 / 2$ at an energy of $1920 \mathrm{MeV}$ with $N a_{0}(980)$ and $N f_{0}(980)$ as its structure. We have also studied the three meson systems $\phi K \bar{K}, \phi \pi \pi$ and found dynamical generation of a resonance with $\phi f_{0}(980)$ structure, $J^{P C}=1^{--}$and a mass around $2150 \mathrm{MeV}$, which can be identified with the $X(2175)$ resonance.

International Workshop on Effective Field Theories: from the pion to the upsilon February 2-6 2009

Valencia, Spain

\footnotetext{
* Speaker.
} 


\section{Introduction}

Two-body meson-baryon and meson-meson systems, as for example, $\bar{K} N, \pi \Sigma, \pi \Lambda, \pi N, \pi \pi$, $K \bar{K}, \pi \eta$, etc., have been studied extensively using unitary chiral dynamics and dynamical generation of many baryon and meson resonances in these systems has been found, for instance, the $\Lambda(1405), \Lambda(1520), N^{*}(1535)$, etc., in the baryon sector and the $f_{0}(980), a_{0}(980), \sigma(600)$, etc., in the meson sector $[1,2,3,4,5,6,7,8,9]$. It has also been found that all these states contain important meson-baryon and meson-meson components, respectively, in their wave functions, which play an important role in the description of their physical properties. Since the interaction in such two-body systems is strongly attractive, it seems plausible to anticipate that the addition of one more meson or baryon could lead to the generation of new states in which case the interaction of the three particles could be determinant in understanding some experimental findings. Some existing resonances, which can be strongly expected to be examples of such situation, are the $N^{*}(1710)$, which decays with $40-90 \%$ branching ratio to $\pi \pi N$ and with $10-40 \%$ for that $\pi \pi N$ system in which the $\pi-\pi$ interaction is in s wave, with isospin zero [10]. The $\Sigma(1660)$, which shows up as a clear signal in the reaction $K^{-} p \rightarrow \pi^{0} \pi^{0} \Sigma^{0}$ [11]. Also, recently, a new meson resonance, $X(2175)$, has been claimed by BABAR and BES $[12,13,14]$ in the $e^{+} e^{-} \rightarrow \phi f_{0}(980)$ and $J / \psi \rightarrow \eta \phi f_{0}(980)$ reactions with quantum numbers $1^{--}$. Therefore, a study of two meson and one baryon systems, like $\pi \pi N, \pi \pi \Sigma, \pi \bar{K} N$, etc., and three-meson systems like, $\phi \pi \pi, \phi K \bar{K}, K^{*} \pi K$, etc., could shed light in the understanding of the properties of states like the $N^{*}(1710), \Sigma(1660), X(2175)$, etc.

With this motivation, we have extended the two-body chiral studies to those three-body systems in which the Faddeev equations are solved using, as input, the two-body chiral $t$-matrices which are calculated by solving the Bethe-Salpeter equation in the coupled channel approach for each of the different pairs of the three-body system.

\section{The Framework}

In order to get the three-body $T$ matrix we follow the procedure developed in $[15,16,17,18]$. In this approach, due to the fact that the chiral $t$-matrices can be explicitly decomposed into a term depending on the invariant mass of the interacting pair and an off-shell part which is inversely proportional to a propagator, an explicit cancellation between the contribution of the off-shell part of the $t$-matrices with a three-body contact term originated from the chiral Lagrangian is found. This converts the integral Faddeev equations into the following algebraic ones

$$
\begin{aligned}
& T_{R}^{12}=t^{1} g^{12} t^{2}+t^{1}\left[G^{121} T_{R}^{21}+G^{123} T_{R}^{23}\right] \\
& T_{R}^{13}=t^{1} g^{13} t^{3}+t^{1}\left[G^{131} T_{R}^{31}+G^{132} T_{R}^{32}\right] \\
& T_{R}^{21}=t^{2} g^{21} t^{1}+t^{2}\left[G^{212} T_{R}^{12}+G^{213} T_{R}^{13}\right] \\
& T_{R}^{23}=t^{2} g^{23} t^{3}+t^{2}\left[G^{231} T_{R}^{31}+G^{232} T_{R}^{32}\right] \\
& T_{R}^{31}=t^{3} g^{31} t^{1}+t^{3}\left[G^{312} T_{R}^{12}+G^{313} T_{R}^{13}\right] \\
& T_{R}^{32}=t^{3} g^{32} t^{2}+t^{3}\left[G^{321} T_{R}^{21}+G^{323} T_{R}^{23}\right]
\end{aligned}
$$


The $T_{R}^{i j}$ partitions include all the different contributions to the three-body $T$ - matrix in which the last interactions are given in terms of the two-body $t$-matrices $t^{j}$ and $t^{i}$, respectively. The $T_{R}^{i j}$ matrices are connected to the Faddeev partitions $T^{i}$ through

$$
T^{i}=t^{i} \delta^{3}\left(\vec{k}_{i}^{\prime}-\vec{k}_{i}\right)+\sum_{j \neq i=1}^{3} T_{R}^{i j}, \quad i=1,2,3
$$

with $\vec{k}_{i}\left(\vec{k}_{i}^{\prime}\right)$ being the initial (final) momentum of the particle $i$. Therefore, the full three-body $T$-matrix is expressed in terms of $T_{R}^{i j}$ as

$$
\begin{aligned}
T & =\sum_{i=1}^{3} T^{i}=\sum_{i=1}^{3} t^{i} \delta^{3}\left(\vec{k}_{i}^{\prime}-\vec{k}_{i}\right)+T_{R} \\
T_{R} & \equiv \sum_{i=1}^{3} \sum_{j \neq i=1}^{3} T_{R}^{i j}
\end{aligned}
$$

In Eq.(2.1), $g^{i j}$ is the three-body Green's function of the system and $G^{i j k}$ is a loop function of threeparticles (for more details see $[15,16,17,18]$ ). All the matrices involved in Eq.(2.1) are projected in $\mathrm{S}$-wave, thus giving total $J^{\pi}=1 / 2^{+}$.

\section{Results}

\subsection{Two mesons and one baryon with $S=-1$}

It is well known that the $\pi \Sigma$ system and its coupled channels generate dynamically the two $\Lambda(1405) S_{01}$ resonances [1,6]. If we add, for example, one more pion, we will end up with a system in which the interaction between the different pairs is attractive in some energy range, in addition to the attractive interaction in the $\pi \Sigma$ region, the two pion interaction is also strongly attractive in nature around the $\sigma(600)$ and $f_{0}(980)$ mass $[3,7]$. Thus, in some energy range there will be attractive interaction between the three pairs. This means that systems as $\pi \pi \Sigma, \pi \pi \Lambda$, etc., can get bound through the mutual interaction between the three particles. In order to build the coupled channels, we take all the mesons from the pseudoscalar octet and couple with the baryons from the $1 / 2^{+}$octet to obtain total strangeness -1 . To the resulting system we add a pion which leads to twenty-two coupled channels for total charge of the three particles equal to zero [15]. Eqs.(2.1) have been solved using all these coupled channels and dynamical generation of six resonances has been found with the properties of $\Sigma(1770), \Sigma(1660), \Sigma(1620), \Sigma(1560), \Lambda(1810)$ and $\Lambda(1600)$, respectively.

\subsection{Two mesons and one baryon with $S=0$}

We solve Eqs.(2.1) for the $\pi \pi N$ system for total charge zero considering fourteen coupled channels $[17,18]$. The calculated three-body $T$-matrix reveals dynamical generation of four resonances: (1) One with mass around $1704 \mathrm{MeV}$ and $375 \mathrm{MeV}$ of width, which we obtain when the two pions interact for an invariant mass close to $600 \mathrm{MeV}$ in isospin zero, thus, in the $\sigma(600)$ region. This is in perfect agreement with the feature of the $N^{*}(1710)$ listed by the PDG [10]. (2) 
Another one with mass around that of the $N^{*}(2100)$, with one of the $\pi N$ pairs simultaneously resonating as the $N^{*}(1650)$, implying a $\pi N^{*}(1650)$ structure. (3) Another one with mass and quantum numbers of the $\Delta(1910)$. (4) And yet another peak around $1920 \mathrm{MeV}$ shows up in the $T$-matrix for the $K \bar{K} N$ channel with total isospin $1 / 2, J^{\pi}=1 / 2^{+}$, when the $K \bar{K}$ system clusters to generate the $f_{0}(980)$ and the $a_{0}(980)$ resonances. This state is not listed in the PDG and it was already suggested by the authors of [19]. In fact, there are some experimental findings which favor the existence of a $N^{*}$ with spin-parity $1 / 2^{+}$at an energy close to $1910 \mathrm{MeV}$, although better experimental studies are needed to confirm this state.

\subsection{Three mesons with $S=0$}

A study of the $e^{+} e^{-} \rightarrow \phi f_{0}(980)$ reaction was made through a loop mechanism involving pseudoscalar and vector meson loops [20]. The authors of [20] could explain the background observed by different experimental groups $[12,13,14]$ for that reaction, but they could not explain the experimental peak obtained around an energy of $2175 \mathrm{MeV}$ in the invariant mass of $\phi f_{0}(980)$ $[12,13,14]$. In the chiral models, the $f_{0}(980)$ resonance appears in the $K \bar{K}$ interaction with a strong coupling to that channel [3]. Therefore, a study of the $\phi K \bar{K}$ system could explain the experimental results. In [16] we solved Eqs.(2.1) taking as coupled channels $\phi K \bar{K}$ and $\phi \pi \pi$ with total charge zero. We obtain a peak around $2150 \mathrm{MeV}$ with a width of $27 \mathrm{MeV}$ when the $K \bar{K}$ and $\pi \pi$ system generate dynamically the $f_{0}(980)$, thus, confirming the experimental finding. We implemented the result of [20] as the final state interaction for the $e^{+} e^{-} \rightarrow \phi f_{0}(980)$ and calculated the cross section for that process. The result is a peak in the cross section around $2175 \mathrm{MeV}$ which is in agreement with the experimental cross section obtained by different experimental groups [12, 13, 14].

\section{Conclusions}

Using unitary chiral dynamics, we have developed a formalism where the integral Faddeev equations become algebraic ones and which can be solved by inverting matrices. We have used this formalism to study different three-body systems, concretely, to the $\pi \bar{K} N$ system and its coupled channels which resulted into dynamical generation of four $\Sigma$ 's and two $\Lambda^{\prime}$ 's with $J^{P}=1 / 2^{+}$, which can be identified with the low lying $1 / 2^{+} \Sigma$ and $\Lambda$ states listed in the PDG in the energy region 1500$1800 \mathrm{MeV}$. We also investigated the $\pi \pi N$ system and we found two $N^{*}$, s and one $\Delta$ corresponding to the $N^{*}(1710), N^{*}(2100)$ and $\Delta(1910)$, respectively, and a new $N^{*}$ state with a mass close to $1920 \mathrm{MeV}$ and $J^{P}=1 / 2^{+}$. In the meson sector, we have studied the $\phi K \bar{K}$ and $\phi \pi \pi$ systems getting the dynamical generation of the $X(2175)$ resonance, which was observed by several experimental groups.

\section{Acknowledgments}

This work is partly supported by DGICYT contract number FIS2006-03438. We acknowledge the support of the European Community-Research Infrastructure Integrating Activity "Study of Strongly Interacting Matter" (acronym HadronPhysics2, Grant Agreement n. 227431) under the Seventh Framework Programme of EU. K. P. Khemchandani thanks the support by the Fundação 
para a Ciência e a Tecnologia of the Ministério da Ciência, Tecnologia e Ensino Superior of Portugal (SFRH/BPD/40309/2007). A. M. T is supported by a FPU grant of the Ministerio de Ciencia e Innovación.

\section{References}

[1] E. Oset and A. Ramos, Nucl. Phys. A 635, 99 (1998)

[2] T. Inoue, E. Oset and M. J. Vicente Vacas, Phys. Rev. C 65, 035204 (2002)

[3] J. A. Oller and E. Oset, Nucl. Phys. A 620, 438 (1997) [Erratum-ibid. A 652, 407 (1999)]

[4] J. Nieves and E. Ruiz Arriola, Nucl. Phys. A 679, 57 (2000)

[5] B. Borasoy, R. Nissler and W. Weise, Eur. Phys. J. A 25, 79 (2005)

[6] D. Jido, J. A. Oller, E. Oset, A. Ramos and U. G. Meissner, Nucl. Phys. A 725, 181 (2003)

[7] J. A. Oller, E. Oset and J. R. Pelaez, Phys. Rev. Lett. 80, 3452 (1998).

[8] V. Bernard, N. Kaiser and U. G. Meissner, Phys. Rev. C 52, 2185 (1995).

[9] L. Roca, S. Sarkar, V. K. Magas and E. Oset, Phys. Rev. C 73, 045208 (2006).

[10] C. Amsler et al. (Particle Data Group), Physics Letters B667, 1 (2008) .

[11] S. Prakhov et al. [Crystall Ball Collaboration], Phys. Rev. C 70, 034605 (2004).

[12] B. Aubert et al. [BABAR Collaboration], Phys. Rev. D 74, 091103 (2006).

[13] B. Aubert et al. [BABAR Collaboration], Phys. Rev. D 76, 012008 (2007).

[14] M. Ablikim et al. [BES Collaboration], Phys. Rev. Lett. 100, 102003 (2008).

[15] A. Martinez Torres, K. P. Khemchandani and E. Oset, Phys. Rev. C 77, 042203 (2008).

[16] A. Martinez Torres, K. P. Khemchandani, L. S. Geng, M. Napsuciale and E. Oset, Phys. Rev. D 78, 074031 (2008).

[17] K. P. Khemchandani, A. Martinez Torres and E. Oset, Eur. Phys. J. A 37, 233 (2008).

[18] A. Martinez Torres, K. P. Khemchandani and E. Oset, arXiv:0812.2235 [nucl-th].

[19] D. Jido and Y. Kanada-En'yo, Phys. Rev. C 78, 035203 (2008).

[20] M. Napsuciale, E. Oset, K. Sasaki and C. A. Vaquera-Araujo, Phys. Rev. D 76, 074012 (2007). 